\title{
Performance of a universal prenatal screening program incorporating cell-free fetal DNA analysis in Ontario, Canada
}

\author{
Shelley D. Dougan MSc, Nan Okun MD MHSc, Kara Bellai-Dussault MSc, Lynn Meng MSc, Heather E. Howley MSc, \\ Tianhua Huang PhD, Jessica Reszel RN MScN, Andrea Lanes PhD, Mark C. Walker MD MSc, \\ Christine M. Armour MSc MD
}

Cite as: CMAJ 2021 August 3;193:E1156-63. doi: 10.1503/cmaj.202456

\begin{abstract}
BACKGROUND: The emergence of cellfree fetal DNA (cfDNA) testing technology has disrupted the landscape of prenatal screening for trisomies 21 (T21) and 18 (T18). Publicly funded systems around the world are grappling with how to best integrate this more accurate but costly technology, as there is limited evidence about its incremental value in real-world conditions. The objectives of this study were to describe the population-based performance of Ontario's prenatal screening program, which incorporates publicly funded cfDNA screening for specific indications, and the effect of cfDNA testing on the screening and diagnostic choices made by pregnant people.
\end{abstract}

METHODS: We conducted a retrospective, descriptive cohort study using routinely collected data from Better
Outcomes \& Registry Network (BORN) Ontario, which captures linked population data for prenatal and neonatal health encounters across Ontario. We included all singleton pregnancies with an estimated due date between Sept. 1, 2016, and Mar. 31, 2019, that underwent publicly funded prenatal screening in Ontario, and a comparison cohort from Apr. 1, 2012, and Mar. 31, 2013. We assessed performance of the screening program for the detection of T21 or T18 by calculating sensitivity, specificity, positive predictive value and negative predictive value against diagnostic cytogenetic results or birth outcomes. We assessed the impact of the program by calculating the proportion of T21 screen-positive pregnancies undergoing subsequent cfDNA screening and invasive prenatal diagnostic testing.
RESULTS: The study cohort included 373682 pregnancies. The prenatal screening program had an uptake of $69.9 \%$, a screen-positive rate and sensitivity of $1.6 \%$ and $89.9 \%$ for $\mathrm{T} 21$, and $0.2 \%$ and $80.5 \%$ for T18, respectively. The test failure rate for cfDNA screening was $2.2 \%$. Invasive prenatal diagnostic testing decreased from $4.4 \%$ in $2012-2013$ to $2.4 \%$ over the study period; $65.2 \%$ of pregnant people who received a screen-positive result from cfDNA testing went on to have invasive prenatal diagnostic testing.

INTERPRETATION: This publicly funded screening program, incorporating cfDNA analysis for common aneuploidies, showed robust performance, a substantial reduction in invasive prenatal diagnostic testing and that pregnant people exercise autonomy in their choices about prenatal screening and diagnosis.

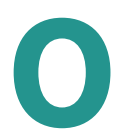

ffering screening for chromosomal aneuploidies, including trisomies 21 (T21) and 18 (T18), is part of routine prenatal care. To this end, multiple marker screening, which uses a combination of ultrasound and maternal serum biomarkers, has been publicly funded in Ontario since the 1990s (Appendix 1 , Table S1, available at www.cmaj.ca/lookup/doi/10.1503/ cmaj.202456/tab-related-content). ${ }^{1}$ In 2012, private diagnostic laboratories began marketing cell-free fetal DNA (cfDNA) testing across Canada. Also known as noninvasive prenatal testing, cfDNA analysis is a maternal blood test that analyzes fetal DNA that originates in the placenta. This disruptive technology shows better detection of fetal aneuploidies with fewer false-positive results than multiple marker screening, ${ }^{2}$ and its adoption has led to fewer invasive procedures for prenatal diagnosis.,4 Market forces and concerns over equitable access led to rapid and variable adoption of cfDNA analysis into prenatal screening programs across Canada and internationally. ${ }^{4-7}$ The current price (about Can $\left.\$ 390\right)^{8}$ of cfDNA analysis precludes it from being publicly funded as a universal test. Instead, publicly funded programs often use a contingent model, offering cfDNA screening for pregnancies with specific indicators that increase the likelihood of aneuploidy. ${ }^{9}$ However, indications for accessing cfDNA screening vary widely across jurisdictions. ${ }^{10,11}$ 
Since 2016, publicly funded cfDNA screening has been offered in Ontario for the following specific indications: a screen-positive result from multiple marker screening, maternal age $>40$ years at estimated due date, certain concerning findings on fetal ultrasound or a previous pregnancy with aneuploidy (Figure 1).

Prenatal Screening Ontario (www.prenatalscreeningontario.ca) is a government-funded program that coordinates provincial prenatal screening, facilitates the incorporation of evolving technologies and screening options, and is responsible for ongoing quality assurance reporting. Their policy planning is informed by data from Ontario's perinatal registry, Better Outcomes Registry \& Network (BORN) Ontario (www.bornontario.ca) (Appendix 1, Supplemental Figure S1). Evaluating the effect of new technologies (e.g., test accuracy, downstream testing, patient choice) is critical to screening programs ${ }^{13}$ and depends on rigorously collected and analyzed data. Previously published analyses from other multiple marker or contingent cfDNA screening programs are limited by a lack of population-level data linking prenatal screening results with cytogenetic records or birth outcomes. ${ }^{3,14-17}$

Our primary objective for this study was to report on the overall and modality-specific performance of Ontario's prenatal screening program, wherein the offer of cfDNA screening is contingent on specific criteria that increase the likelihood of T21 or T18 (Figure 1). Our secondary objective was to report on the impact of this screening approach on test utilization by pregnant individuals.

\section{Methods}

\section{Study design and data source}

We conducted a retrospective, population-based, descriptive cohort study that used routinely collected data within BORN. Established in 2009, BORN is a prescribed registry under the Personal Health Information Protection $A c t^{18}$ that collects critical health data about every pregnancy, birth and newborn in Ontario directly from fertility clinics, screening and diagnostic laboratories, hospitals, midwifery practice groups and other organizations across the province. ${ }^{19}$ All publicly funded multiple marker and cfDNA screening is performed by Ontario-based laboratories that contribute complete testing data to BORN. This registry currently holds data for more than 1.4 million mother-newborn dyads, including linkable population data on all prenatal screening modalities, pre- and postnatal cytogenetic results, and birth outcomes (i.e., born live or deceased, suspected or confirmed congenital anomalies)..$^{20}$ Details about BORN's data quality, availability, cleaning and linkage have been previously described. ${ }^{20}$

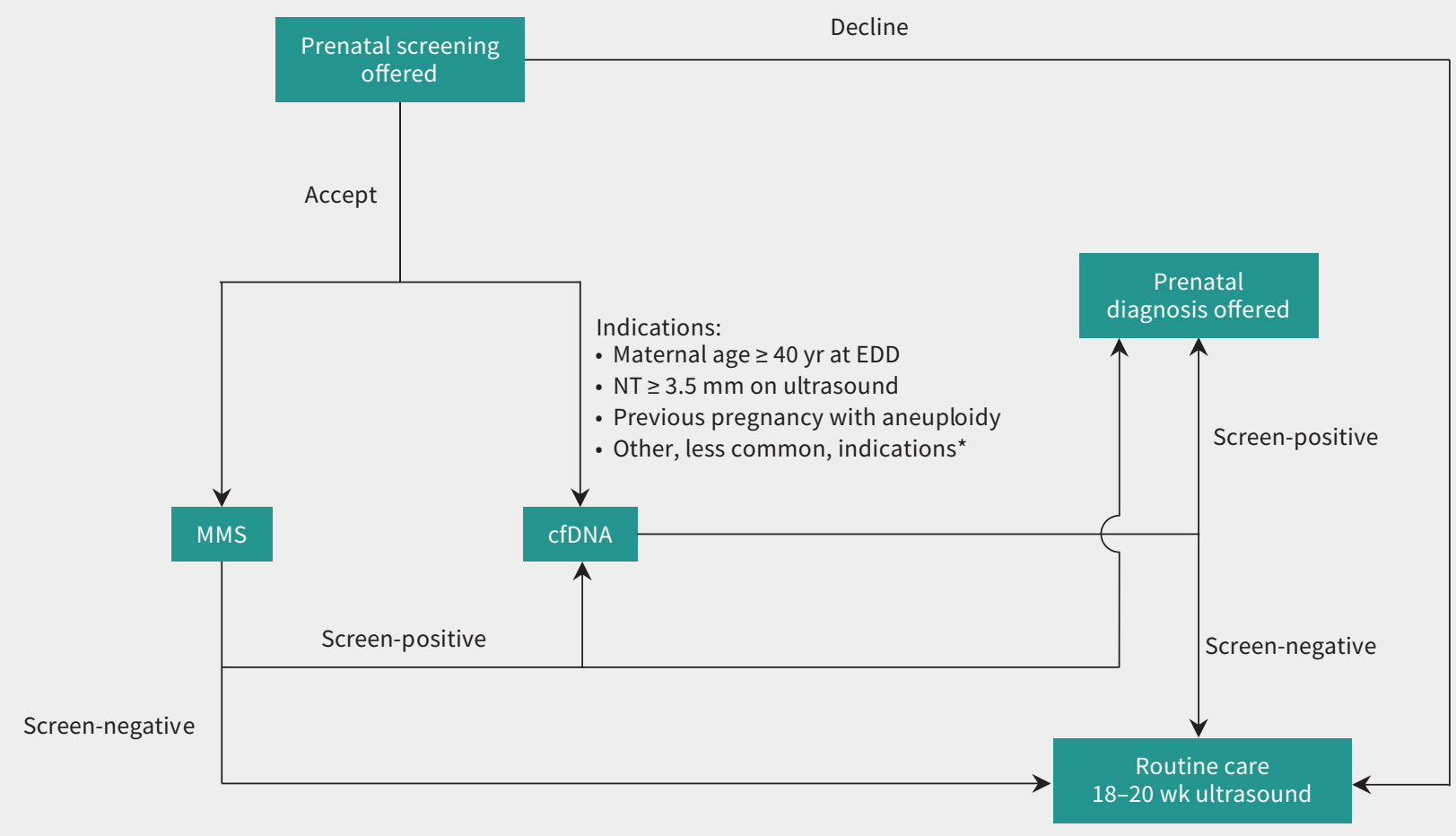

Figure 1: Ontario's universal and publicly funded model for incorporating cfDNA analysis into prenatal screening for aneuploidy. In 2016, Ontario began funding cfDNA prenatal screening for the common autosomal aneuploidies (e.g., trisomy 21, 18). Pregnant patients at high risk for fetal aneuploidy are eligible for publicly funded cfDNA screening after a screen-positive MMS (defined as risk $\geq 1$ in 350 for the enhanced first trimester screening test or risk $\geq 1$ in 200 for the quadruple marker screening test) or as a first-tier screen. Criteria for determining eligibility for first-tier cfDNA screening are based on recommendations from the provincial advisory group, with the goal of optimizing performance and containing costs. ${ }^{12} \mathrm{Although}$ presented as a flowchart, this figure does not represent chronological care pathways or a stepwise approach; in the real world, decisions about screening and testing do not always follow a model. Note: cfDNA = cell-free fetal DNA screening, EDD = estimated due date, MMS = multiple marker screening, PND = prenatal diagnostic testing, NT = nuchal translucency. ${ }^{*}$ See Prenatal Screening Ontario's website for all indications for first-tier cfDNA screening (https://prenatalscreeningontario.ca/en/pso/about-prenatal-screening/nipt-funding-criteria.aspx). 


\section{Inclusion and exclusion criteria}

We included all singleton pregnancies in Ontario with an estimated due date between Sept. 1, 2016, and Mar. 31, 2019, that underwent publicly funded prenatal screening. We excluded records from selffunded cfDNA screening as these fall outside the scope of our public screening system. We deterministically linked patient postal codes to Census data to obtain neighbourhood income quintiles.

We also included a cohort of singleton pregnancies with an estimated due date between Apr. 1, 2012, and Mar. 31, 2013, to evaluate the effect of cfDNA screening on uptake of prenatal diagnostic testing. We chose this timeframe as cfDNA screening became sporadically available in the latter part of 2013.

\section{Outcome measures and construction of cohorts}

To meet our primary objective, we calculated the sensitivity (detection rate), specificity, and screen-positive rate of Ontario's prenatal screening system for T21 and T18. A challenge for ascertaining the T21 and T18 status of all pregnancies that underwent prenatal screening is that diagnosis of aneuploidy via cytogenetic testing is performed only for a small subset of pregnancies and infants with appropriate clinical indications. Thus, we used BORN registry data for pregnancies without diagnostic results. Infants (and linked pregnancies) were presumed unaffected when birth records showed that neither T21 nor T18 were identified by, at minimum, 3 months of age. To form binary classification tables, we constructed 6 performance analysis cohorts from this data set to evaluate overall and modality-specific (multiple marker or cfDNA) screening for either T18 or T21. We excluded records from performance analysis cohorts for any of the following reasons: a screening record with no result; a cytogenetic result designated as mosaic, partial, uninterpretable or inconclusive; or a record with no associated abnormal or normal cytogenetic outcome and no negative birth outcome. Exclusion criteria were not mutually exclusive.

To meet our secondary objective we calculated the uptake of prenatal screening for T21, the number of pregnancies that were screen-positive using multiple marker screening that underwent either cfDNA screening or invasive prenatal diagnostic testing, and the number of pregnancies that were screen-positive using cfDNA screening that underwent prenatal diagnostic testing. We also compared the rate of screened pregnancies that underwent prenatal diagnostic testing of our main cohort with our 2012-2013 cohort, expressed as a proportion of all screened pregnancies in Ontario.

\section{Statistical analyses}

We summarized demographic data using means and standard deviations or counts and percentages, where appropriate. We calculated Clopper-Pearson confidence intervals for the performance measures. We extracted data from the source database using SAS software version 9.4 and prepared them for analysis and analyzed using $\mathrm{R}$ version 3.5.2.

\section{Ethics approval}

This study was approved by the research ethics boards of Children's Hospital of Eastern Ontario (protocol 19/06PE), Ottawa Health Sciences Network (protocol 20190482-01H) and Mount Sinai Hospital (protocol 19-0181-C).

\section{Results}

\section{Participants and demographics}

Over the study period, we analyzed 373682 eligible singleton pregnancies with access to a variety of screening modalities (Appendix 1, Supplemental Figure S2). Pregnant people who had first-tier cfDNA screening were older (as expected as per eligibility criteria) and had a higher income than those those who had either multiple marker screening alone or multiple marker and cfDNA screening. Table 1 describes maternal characteristics, and stratifies the number of pregnancies by screening modality.

\section{Screening performance}

Prenatal screening for either T21 or T18 was performed in 261096 (69.9\%) of singleton pregnancies. Not every pregnancy had a screening result returned for both T21 and T18; record inclusion and exclusion for each performance analysis cohort is illustrated in Figure 2. Of all screened pregnancies, 97.8\% had multiple marker screening, of which $96.1 \%$ were included in our performance analysis for both T21 and T18. Of 22558 cfDNA screening records, $93.6 \%$ were included in our analysis for both T21 and T18. The overall analyses of all 261096 pregnancies with either multiple marker or cfDNA screening included 250594 (96.0\%) records for T21 and 250600 (96.0\%) for T18.

The overall sensitivity (detection rate) of our contingent prenatal screening program was $89.94 \%$ for T21 and $80.47 \%$ for T18 (Table 2). Of all pregnancies that had been screened, 4216 (1.6\%) had screen-positive results for T21 and 639 (0.2\%) had screen-positive results for T18, where cfDNA screening results are considered definitive. Overall system specificity was $98.76 \%$ for T21 and $99.89 \%$ for T18, with a negative predictive value of $>99.9 \%$ for both T21 and T18. Given privacy restrictions inherent with the use of registry data, we are unable to provide counts when the cell sizes are fewer than 6 , and therefore cannot report the number of false-negative results.

Of all pregnancies that had been screened, 14091 (5.4\%) pregnancies had an unknown outcome (i.e., they lacked both a follow-up cytogenetic test result and birth outcome) (Figure 2), of which 668 (4.7\%) were screen-positive for T21.

\section{Impact of cfDNA screening on follow-up testing}

We focused on T21 when evaluating the different prenatal screening and diagnostic testing options pursued after a screen-positive result (Figure 3). For 13396 pregnancies with a screen-positive result from multiple marker screening, 1953 (14.6\%) had no further testing and 11443 (85.4\%) underwent follow-up testing. Of the latter, 9901 (86.5\%) had cfDNA screening and 1542 (13.5\%) went directly to prenatal diagnostic testing. Of the 372 pregnancies that were screen-positive after both cfDNA and multiple marker screening, 261 (70.2\%) had invasive prenatal diagnostic testing. Of 159 pregnancies with a positive result after first-tier cfDNA screening, 81 (50.9\%) had prenatal diagnostic testing. Overall uptake of prenatal diagnostic testing after a cfDNA screen-positive result was $65.2 \%$. 
We observed a $45 \%$ reduction in invasive prenatal diagnostic testing since the integration of cfDNA screening; 6242 (2.4\%) of all 261096 screened pregnancies in our main cohort underwent prenatal diagnostic testing, compared with 4208 (4.4\%) of 96501 screened pregnancies in the 2012-2013 cohort that predates the introduction of cfDNA screening.

\section{Interpretation}

Our study provides a large, population-based performance analysis of a contingent cfDNA prenatal screening system, leveraging BORN's perinatal data set. ${ }^{20}$ From a population of 373682 pregnancies, we report an overall uptake of $69.9 \%$, a screen-positive rate and sensitivity of $1.6 \%$ and $89.9 \%$ for $\mathrm{T} 21$, and $0.2 \%$ and $80.5 \%$ for T18, respectively. In agreement with other publications, ${ }^{21,22}$ our data show a cfDNA screening sensitivity of $99.8 \%$ for T21 and $94.4 \%$ for T18, with a test failure rate of $2.2 \%$ (including multiple attempts). Importantly, we observed a twofold reduction (56\%) in invasive prenatal diagnostic testing since the integration of cfDNA screening, which is consistent with other publications. ${ }^{3,4,23}$ Ontario's prenatal screening system was designed based on modelled scenarios ${ }^{12}$ and was intended to align with optimal population-based screening principles; ${ }^{13}$ namely, to provide equitable access to cfDNA tests, to optimize detection and to reduce the overall screen-positive rate without substantially increasing costs. A 2014 cost and performance analysis of 8 modelled screening scenarios showed that integrating cfDNA as a contingent test improved the detection of T21 and reduced prenatal diagnostic testing with a modest increase in costs. ${ }^{12}$ Compared with this modelled scenario, ${ }^{12}$ we observed a slight increase in uptake (70\% v. $67 \%)$ and similar performance.

Our study extends previous research by offering a fully linked, population-based performance assessment of a contingent cfDNA prenatal screening system in Ontario, Canada's most populous province. A key strength of our approach was using linked BORN data to determine the T21 and T18 status of all pregnancies undergoing prenatal screening, which allowed us to identify true negative cases and report on specificity and negative predictive value. A 2018 study from the Danish Fetal Medicine Database reported the performance of a prenatal screening system with contingent cfDNA analysis similar to Ontario's, but the Danish cohort was hospital based, far smaller $(n=6449)$ and more homogeneous. ${ }^{24}$ Compared with the Danish system, which uses lower risk cutoffs for multiple marker screening ${ }^{25}$ to achieve its superior

Table 1: Maternal demographics of singleton pregnancies in Ontario with an estimated due date between Sept. 1, 2016, and Mar. 31, 2019, excluding those with a self-paid screening test

\begin{tabular}{|c|c|c|c|c|}
\hline \multirow[b]{2}{*}{ Characteristics } & \multicolumn{4}{|c|}{ No. $(\%)$ of patients* } \\
\hline & $\begin{array}{c}\text { MMS only } \\
n=238538\end{array}$ & $\begin{array}{c}\text { cfDNA only } \\
n=5750\end{array}$ & $\begin{array}{c}\text { MMS + cfDNA } \\
n=16808\end{array}$ & $\begin{array}{c}\text { No screening } \\
n=112586\end{array}$ \\
\hline Maternal age at EDD, mean \pm SD & $31.0 \pm 4.7$ & $38.9 \pm 5.3$ & $36.0 \pm 5.2$ & $30.4 \pm 5.5$ \\
\hline $\mathrm{GA}$, wk, at MMS screening, mean $\pm \mathrm{SD}$ & $12.7 \pm 1.1$ & NA & $12.70 \pm 0.9$ & NA \\
\hline $\mathrm{GA}$, wk, at cfDNA testing, mean $\pm \mathrm{SD}$ & NA & $13.7 \pm 1.4$ & $15.6 \pm 4.1$ & NA \\
\hline \multicolumn{5}{|l|}{ Maternal BMI } \\
\hline$<18.5$ & $10536(4.4)$ & $175(3.0)$ & $584(3.5)$ & $5360(4.8)$ \\
\hline $18.5-25$ & $104428(43.8)$ & $2215(38.5)$ & $7095(42.2)$ & $47226(41.9)$ \\
\hline $25-30$ & $48570(20.4)$ & $1085(18.9)$ & $3433(20.4)$ & $23351(20.7)$ \\
\hline$\geq 30$ & $37276(15.6)$ & $908(15.8)$ & $2624(15.6)$ & $19353(17.2)$ \\
\hline Missing data & $37728(15.8)$ & $1367(23.8)$ & $3072(18.3)$ & $17296(15.4)$ \\
\hline \multicolumn{5}{|l|}{ Smoking } \\
\hline Any & $12036(5.0)$ & $211(3.7)$ & $540(3.2)$ & $11893(10.6)$ \\
\hline None & $211004(88.5)$ & $4617(80.3)$ & $14763(87.8)$ & $92682(82.3)$ \\
\hline Missing data & 15498 (6.5) & $922(16.0)$ & $1505(9.0)$ & $8011(7.1)$ \\
\hline \multicolumn{5}{|l|}{ Income quintile } \\
\hline Q1 (lowest) & $51650(21.7)$ & $1061(18.5)$ & 3272 (19.5) & 27439 (24.4) \\
\hline Q2 & $48620(20.4)$ & $1092(19.0)$ & 3183 (18.9) & 22267 (19.8) \\
\hline Q3 & $50106(21.0)$ & $1094(19.0)$ & 3425 (20.4) & $21121(18.8)$ \\
\hline Q4 & $47140(19.8)$ & $1123(19.5)$ & $3648(21.7)$ & $18626(16.5)$ \\
\hline Q5 (highest) & 36891 (15.5) & $1260(21.9)$ & 3074 (18.3) & $15152(13.5)$ \\
\hline Missing data & $4131(1.7)$ & $120(2.1)$ & $206(1.2)$ & $7981(7.1)$ \\
\hline
\end{tabular}


Excluded $n=128320$

- MMS not performed $n=118336$

- No MMS result for T21 $n=1647$

- Ineligible cyto result* $n=89$

- No cyto + no negative birth outcome $n=14091$

\section{MMS, T21 analysis} $n=245362$
Excluded $n=128315$

- MMS not performed $n=118336$

- No MMS result for T18 $n=1664$

- Ineligible cyto result* $n=96$

- No cyto + no negative birth outcome $n=14091$

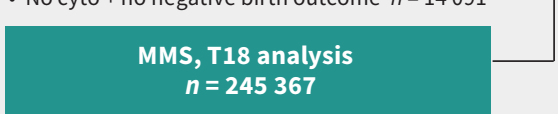

Excluded $n=352570$

- Funded cfDNA testing not performed $n=351124$

- No call result for T21 $n=579$

- Ineligible cyto result* $n=89$

- Nocyto + no negative birth outcome $n=14091$

\section{cfDNA, T21 analysis}

$n=21112$
Excluded multiple births (e.g., twins) $n=6022$

Singleton pregnancies between Sept. 1, 2016, and Mar. 31, 2019 $n=373682$

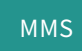

$\mathrm{T} 21$
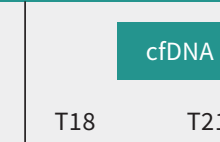
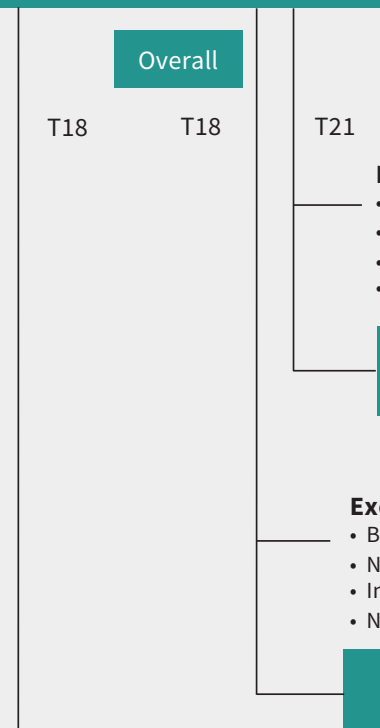

Excluded $n=128088$

- Both not performed $n=112586$

- No results for T21†

- Ineligible cyto result* $n=89$

- No cyto + no negative birth outcome $n=14091$

\section{Overall, T21 analysis} $n=250594$

Excluded $n=123082$

- Both not performed $n=112856$

- No results for T18 $n=6$

- Ineligible cyto result* $n=96$

- No cyto + no negative birth outcome $n=14091$

Overall, T18 analysis $n=250600$

Excluded $n=352567$

- Funded cfDNA testing not performed $n=351124$

- No call result for T18 $n=579$

- Ineligible cyto result* $n=96$

- No cyto + no negative birth outcome $n=14091$

cfDNA, T18 analysis $n=21115$

Figure 2: Construction of performance analysis cohorts. We applied eligibility criteria to all singleton pregnancies between Sept. 1, 2016, and Mar. 31, 2019, to construct 6 cohorts for analysis of overall and modality-specific (multiple marker or cfDNA) screening for either T18 or T21. Reasons for exclusion were not mutually exclusive and individual pregnancies may be represented in multiple cohorts. Note: cfDNA: cell-free fetal DNA, cyto = cytogenetic testing, MMS = multiple marker screening, T18 = trisomy $18, \mathrm{~T} 21=$ trisomy $21 .{ }^{*}$ Ineligible cytogenetic results were those designated as mosaic, partial, uninterpretable or inconclusive. †Small numbers $(n<6)$ were suppressed.

Table 2: Overall and modality-specific performance of a universal and publicly funded prenatal screening program for trisomies 21 (T21) and 18 (T18)

\begin{tabular}{|c|c|c|c|c|c|c|}
\hline \multirow[b]{2}{*}{ Variable } & \multicolumn{2}{|c|}{ Overall } & \multicolumn{2}{|c|}{ MMS } & \multicolumn{2}{|c|}{ cfDNA } \\
\hline & T21 & T18 & T21 & T18 & T21 & T18 \\
\hline Sensitivity $(95 \% \mathrm{CI})$ & $\begin{array}{c}89.94 \\
(87.50-92.05)\end{array}$ & $\begin{array}{c}80.47 \\
(74.53-85.54)\end{array}$ & $\begin{array}{c}86.25 \\
(83.19-88.95)\end{array}$ & $\begin{array}{c}76.79 \\
(69.66-82.94)\end{array}$ & $\begin{array}{c}99.78 \\
(98.77-99.99)\end{array}$ & $\begin{array}{c}94.44 \\
(88.30-97.93)\end{array}$ \\
\hline Specificity $(95 \%$ CI) & $\begin{array}{c}98.76 \\
(98.72-98.81)\end{array}$ & $\begin{array}{c}99.89 \\
(99.88-99.91)\end{array}$ & $\begin{array}{c}94.99 \\
(94.91-95.08)\end{array}$ & $\begin{array}{c}99.76 \\
(99.74-99.78)\end{array}$ & $\begin{array}{c}99.81 \\
(99.74-99.87)\end{array}$ & $\begin{array}{c}99.95 \\
(99.91-99.97)\end{array}$ \\
\hline PPV $(95 \% \mathrm{CI})$ & $\begin{array}{c}17.26 \\
(16.06-18.51)\end{array}$ & $\begin{array}{c}39.23 \\
(34.64-43.96)\end{array}$ & $\begin{array}{c}3.94 \\
(3.61-4.29)\end{array}$ & $\begin{array}{c}17.79 \\
(15.08-20.78)\end{array}$ & $\begin{array}{c}91.99 \\
(89.21-94.24)\end{array}$ & $\begin{array}{c}90.27 \\
(83.25-95.04)\end{array}$ \\
\hline NPV (95\% Cl) & $\begin{array}{c}99.97 \\
(99.96-99.98)\end{array}$ & $\begin{array}{c}99.98 \\
(99.98-99.99)\end{array}$ & $\begin{array}{c}99.97 \\
(99.96-99.97)\end{array}$ & $\begin{array}{c}99.98 \\
(99.98-99.99)\end{array}$ & $\begin{array}{c}>99.99 \\
(99.97-100.00)\end{array}$ & $\begin{array}{c}99.97 \\
(99.94-99.99)\end{array}$ \\
\hline
\end{tabular}

Note: $c f D N A=$ cell-free fetal DNA screening, $\mathrm{Cl}=$ confidence interval, $M M S=$ multiple marker screening, NPV = negative predictive value, $\mathrm{PPV}=$ positive predictive value 


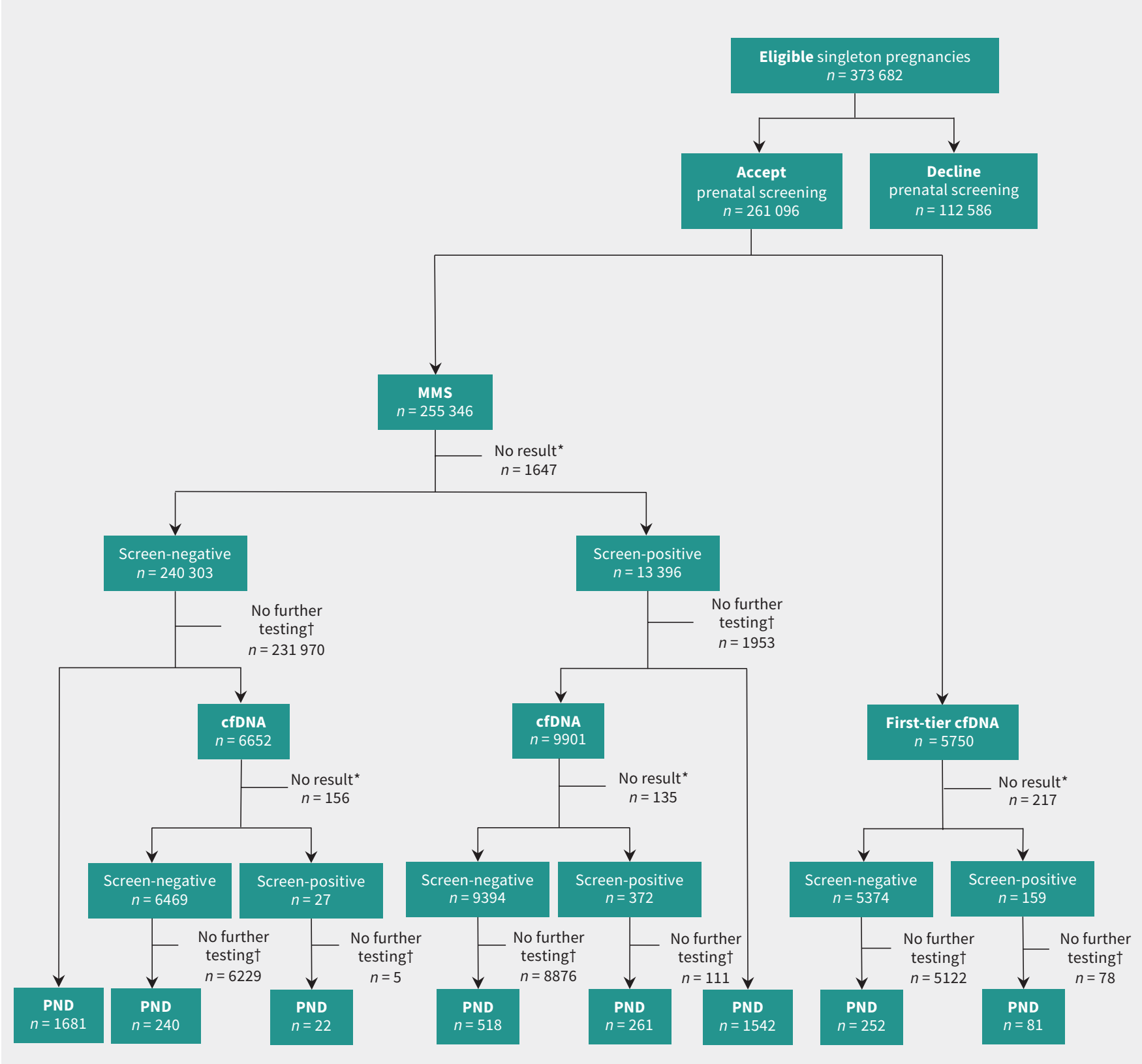

Figure 3: Uptake of screening and invasive prenatal diagnostic testing (PND) for trisomy 21 via Ontario's prenatal screening program. Note: cfDNA = cell-free fetal DNA screening; MMS = multiple marker screening. During the 2.5-year study period, an offer of prenatal screening was accepted for 261096 singleton pregnancies (69.9\%). This flowchart illustrates the variety of screening and testing options pursued by pregnant people in Ontario. Real-world utilization is very different than the ideal model presented in Figure 1. "No result" refers to test failures and includes multiple test attempts. " "No further testing" refers to no further publicly funded cfDNA or invasive testing for aneuploidy.

performance ( $100 \%$ sensitivity with $98.8 \%$ specificity), ${ }^{24}$ we show that a quality screening system with high performance is still attainable with more stringent cutoffs for multiple marker screening. Determining appropriate cutoffs is important in a public system, both to control costs and to avoid potential harms arising from false-positive results; ${ }^{9,26}$ Prenatal Screening Ontario is actively exploring how to optimize this aspect of our system. ${ }^{9,26}$

Routinely collected population data allow us to track the use of different tests as pregnant people navigate the program. Pregnant people may not follow a standard care pathway; some have
cfDNA screening before or at the same time as multiple marker screening. Indications prompting cfDNA screening or prenatal diagnostic testing may occur in spite of a negative result from multiple marker screening, most commonly maternal age $>40$ years or a subsequent ultrasound finding, thus accounting for the uptake of cfDNA screening and prenatal diagnostic testing even with a negative result from multiple marker screening.

The high accuracy and noninvasive nature of cfDNA screening has led to general concerns regarding the routinization of this test and its effect on informed choice..$^{27,28}$ Ontario's pregnant 
population pursued a variety of options after a screen-positive result, consistent with other publications ${ }^{23}$ showing that pregnant people make individual choices. Notably, we observed that although there is considerable uptake of publicly funded cfDNA screening, only $65.2 \%$ of people who received a positive result from cfDNA screening (contingent or first-tier) went on to have invasive prenatal diagnostic testing. Professional organizations uniformly advise diagnostic confirmation via cytogenetic testing before interrupting a pregnancy; ${ }^{29}$ therefore, our data may be interpreted to show that pregnant people are supported in making informed choices, including pregnancy continuation. The high positive predictive value of cfDNA screening allows for informed care to provide the best prenatal and perinatal outcomes after a screen-positive result from cfDNA screening. We observed an almost twofold (45\%) overall reduction in invasive prenatal diagnostic testing since the integration of cfDNA screening, almost certainly because of its very low false-positive rate.

Given the sample size and data quality, our study provides insights for other jurisdictions, and shows that integrating cfDNA screening as a contingent (rather than universal) test can yield excellent performance and reduce unnecessary prenatal diagnostic testing for T21 and T18. We also show the value of comprehensive, registry-based data to monitor and optimize system performance. Using these findings as a baseline and with the power of registry data, Prenatal Screening Ontario can determine the cost-effectiveness of different screening models and algorithms. These data can also rapidly inform evidence-based guidance regarding the integration of new technologies (e.g., new approaches to cfDNA screening for aneuploidy), the detection of other conditions (e.g., microdeletion syndromes, preeclampsia, congenital anomalies) and adjustments for changing conditions (e.g., the impact of COVID-19 restrictions on access to ultrasound for nuchal translucency measurement).

\section{Limitations}

Despite our comprehensive data set, $5.4 \%$ of screened pregnancies were excluded from performance analyses because they had an unknown outcome; this is an inherent limitation of retrospective cohort studies. The proportion of unknown outcomes is similar to the predicted spontaneous pregnancy loss after 12 weeks of about $6 \% \cdot{ }^{30,31}$ Not unexpectedly, we observed substantially more screen-positive results among people with an unknown outcome compared with those with a known outcome $(4.7 \% \mathrm{v}$. $1.6 \%$ ) as pregnancy loss is associated with up to a $70 \%$ chance of aneuploidy; ${ }^{32-34}$ the exclusion of these records likely attenuates the reported performance.

\section{Conclusion}

Previous performance estimates for Ontario's prenatal screening system were based on modelled data. Using robust linkage of prenatal screening results with birth outcomes, including all true negatives, we show that our real system achieves higher uptake and better detection rates than our previous modelling predicted. We also observed a twofold reduction in invasive prenatal diagnostic testing since the integration of cfDNA screening. We illustrated the power of registry data to monitor real-world performance of prenatal screening systems and enable real-time optimization; these data are used to drive continuous quality improvement of our program and to answer important questions about the use and utility of emerging screening methods.

\section{References}

1. Wald NJ, Rodeck C, Hackshaw AK, et al.; SURUSS Research Group. First and second trimester antenatal screening for Down's syndrome: the results of the serum, urine and ultrasound screening study (SURUSS). Health Technol Assess 2003;7:1-77.

2. Allyse M, Minear MA, Berson E, et al. Non-invasive prenatal testing: a review of international implementation and challenges. Int J Womens Health 2015;7: 113-26.

3. Chitty LS, Wright D, Hill M, et al. Uptake, outcomes, and costs of implementing non-invasive prenatal testing for Down's syndrome into NHS maternity care: prospective cohort study in eight diverse maternity units. BMJ 2016;354:i3426.

4. Oepkes D, Page-Christiaens GCL, Bax CJ, et al.; Dutch NIPT Consortium. Trial by Dutch laboratories for evaluation of non-invasive prenatal testing. Part I clinical impact. Prenat Diagn 2016;36:1083-90.

5. Gil MM, Accurti V, Santacruz B, et al. Analysis of cell-free DNA in maternal blood in screening for aneuploidies: updated meta-analysis. Ultrasound Obstet Gynecol 2017;50:302-14.

6. Salomon LJ, Alfirevic Z, Audibert F, et al.; ISUOG Clinical Standards Committee. ISUOG updated consensus statement on the impact of cfDNA aneuploidy testing on screening policies and prenatal ultrasound practice. Ultrasound Obstet Gynecol 2017;49:815-6.

7. Allyse M, Chandrasekharan S. Too much, too soon?: Commercial provision of noninvasive prenatal screening for subchromosomal abnormalities and beyond. Genet Med 2015;17:958-61.

8. Health Quality Ontario. Noninvasive prenatal testing for trisomies 21,18 , and 13, sex chromosome aneuploidies, and microdeletions: a health technology assessment. Ont Health Technol Assess Ser 2019;19:1-166.

9. Walker BS, Jackson BR, Lagrave D, et al. A cost-effectiveness analysis of cell free DNA as a replacement for serum screening for Down syndrome. Prenat Diagn 2015;35:440-6.

10. Non-Invasive Prenatal Testing (NIPT). Vancouver: Perinatal Services BC. Available: http://www.perinatalservicesbc.ca/health-professionals/professional-resources/ screening/prenatal-genetic/non-invasive-prenatal-testing-nipt (accessed 2021 Mar. 15).

11. NHS fetal anomaly screening programme handbook: valid from August 2018. London (UK): Public Health England; 2018. Available: https://assets.publishing. service.gov.uk/government/uploads/system/uploads/attachment_data/file /749742/NHS_fetal_anomaly_screening_programme_handbook_FINAL1.2 _18.10.18.pdf (accessed 2021 May 31).

12. Okun N, Teitelbaum M, Huang $\mathrm{T}$, et al. The price of performance: a cost and performance analysis of the implementation of cell-free fetal DNA testing for Down syndrome in Ontario, Canada. Prenat Diagn 2014;34:350-6.

13. Dobrow MJ, Hagens V, Chafe R, et al. Consolidated principles for screening based on a systematic review and consensus process. CMAJ 2018;190:E422-9.

14. Gil MM, Revello R, Poon LC, et al. Clinical implementation of routine screening for fetal trisomies in the UK NHS: Cell-free DNA test contingent on results from first-trimester combined test. Ultrasound Obstet Gynecol 2016;47:45-52.

15. Kazerouni NN, Currier RJ, Flessel M, et al. Detection rate of quadruple-marker screening determined by clinical follow-up and registry data in the statewide California program, July 2007 to February 2009. Prenat Diagn 2011;31:901-6.

16. Lindquist $A$, Hui L, Poulton $A$, et al. State-wide utilization and performance of traditional and cell-free DNA-based prenatal testing pathways: the Victorian Perinatal Record Linkage (PeRL) study. Ultrasound Obstet Gynecol 2020;56:215-24.

17. Maxwell S, O'Leary P, Dickinson JE, et al. Diagnostic performance and costs of contingent screening models for trisomy 21 incorporating non-invasive prenatal testing. Aust N Z J Obstet Gynaecol 2017;57:432-9.

18. Personal Health Information Protection Act, 2004, S.O. 2004, c. 3, Sched. A. Available: https://www.ontario.ca/laws/statute/04p03\#BK44 (accessed 2021 May 31).

19. Impact report: BORN Ontario 2018-2020. Ottawa: BORN Ontario; 2021. Available: https://www.bornontario.ca/en/publications/resources/Documents/BORN-Ontario -Impact-Report-2018-2020-English-version---accessible.pdf (accessed 2021 May 31). 
20. Murphy MSQ, Fell DB, Sprague AE, et al. Data resource profile: Better Outcomes Registry \& Network (BORN) Ontario. Int J Epidemiol 2021 June 7 [Epub ahead of print]. doi: 10.1093/ije/dyab033.

21. Norton ME, Jacobsson B, Swamy GK, et al. Cell-free DNA analysis for noninvasive examination of trisomy. N Engl J Med 2015;372:1589-97.

22. Pergament E, Cuckle H, Zimmermann B, et al. Single-nucleotide polymorphismbased noninvasive prenatal screening in a high-risk and low-risk cohort. Obstet Gynecol 2014;124:210-8.

23. Hill M, Barrett A, Choolani M, et al. Has noninvasive prenatal testing impacted termination of pregnancy and live birth rates of infants with Down syndrome? Prenat Diagn 2017;37:1281-90.

24. Miltoft CB, Rode L, Ekelund CK, et al. Contingent first-trimester screening for aneuploidies with cell-free DNA in a Danish clinical setting. Ultrasound Obstet Gynecol 2018;51:470-9.

25. Vogel I, Tabor A, Ekelund C, et al.; The Danish Fetal Medicine Study Group, and the Danish Cytogenetic Study Group. Population-based screening for trisomies and atypical chromosomal abnormalities: improving efficacy using the combined first trimester screening algorithm as well as individual risk parameters. Fetal Diagn Ther 2019;45:424-9.

26. Nicolaides $\mathrm{KH}$, Wright $\mathrm{D}$, Poon LC, et al. First-trimester contingent screening for trisomy 21 by biomarkers and maternal blood cell-free DNA testing. Ultrasound Obstet Gynecol 2013;42:41-50
27. Vanstone M, Cernat A, Majid U, et al. Perspectives of pregnant people and clinicians on noninvasive prenatal testing: a systematic review and qualitative meta-synthesis. Ont Health Technol Assess Ser 2019;19:1-38.

28. Lewis $\mathrm{C}$, Hill M, Chitty LS. Offering non-invasive prenatal testing as part of routine clinical service. Can high levels of informed choice be maintained? Prenat Diagn 2017;37:1130-7.

29. Audibert F, De Bie I, Johnson J-A, et al. No. 348-Joint SOGC-CCMG Guideline: update on prenatal screening for fetal aneuploidy, fetal anomalies, and adverse pregnancy outcomes. J Obstet Gynaecol Can 2017;39:805-17.

30. Rossen LM, Ahrens KA, Branum AM. Trends in risk of pregnancy loss among US women, 1990-2011. Paediatr Perinat Epidemiol 2018;32:19-29.

31. Michels TC, Tiu AY. Second trimester pregnancy loss. Am Fam Physician 2007;76: 1341-6.

32. Levy B, Sigurjonsson S, Pettersen B, et al. Genomic imbalance in products of conception: Single-nucleotide polymorphism chromosomal microarray analysis. Obstet Gynecol 2014;124:202-9.

33. Romero ST, Geiersbach KB, Paxton CN, et al. Differentiation of genetic abnormalities in early pregnancy loss. Ultrasound Obstet Gynecol 2015;45:89-94.

34. Soler A, Morales C, Mademont-Soler I, et al. Overview of chromosome abnormalities in first trimester miscarriages: a series of 1,011 consecutive chorionic villi sample karyotypes. Cytogenet Genome Res 2017;152:81-9.
Competing interests: Nan Okun reports funding from Roche Diagnostic, outside the submitted work. No other competing interests were declared.

This article has been peer reviewed.

Affiliations: Prenatal Screening Ontario (Dougan, Okun, Bellai-Dussault, Meng, Huang, Reszel, Lanes, Walker, Armour), Better Outcomes Registry \& Network (BORN) Ontario; Children's Hospital of Eastern Ontario (CHEO) Research Institute (Dougan, Bellai-Dussault, Howley, Reszel, Walker, Armour), Ottawa, Ont.; Mount Sinai Hospital (Okun); Genetics Program (Huang), North York General Hospital; Department of Obstetrics and Gynecology (Huang), University of Toronto, Toronto, Ont.; Department of Obstetrics and Gynecology (Walker), University of Ottawa; Ottawa Hospital Research Institute (OHRI) (Walker); Department of Pediatrics (Armour), University of Ottawa, Ottawa, Ont.

Contributors: Shelley Dougan, Nan Okun, Kara Bellai-Dussault, Jessica Reszel, Andrea Lanes, Mark Walker and Christine Armour conceptualized the study, which Shelley Dougan, Nan Okun, Kara Bellai-Dussault, Lynn Meng, Tianhua Huang and Christine Armour designed. Shelley Dougan, Kara Bellai-Dussault, Lynn Meng, Tianhua Huang and Christine Armour acquired data, which was analyzed and interpreted by Kara Bellai-Dussault, Lynn Meng and Heather Howley. Shelley Dougan, Nan Okun, Kara Bellai-Dussault, Heather Howley, Jessica Reszel, Andrea Lanes and Christine Armour drafted the manuscript. All of the authors revised it critically for important intellectual content, gave final approval of the version to be published and agreed to be accountable for all aspects of the work. Shelley Dougan and Nan Okun are co-first authors.

Content licence: This is an Open Access article distributed in accordance with the terms of the Creative Commons Attribution (CC BY-NC-ND 4.0) licence, which permits use, distribution and reproduction in any medium, provided that the original publication is properly cited, the use is noncommercial (i.e., research or educational use), and no modifications or adaptations are made. See: https://creativecommons.org/licenses/ by-nc-nd/4.0/

Funding: This study was supported in part by BORN Ontario, which is funded by the Ontario Ministry of Health. Heather Howley is supported by the CHEO Foundation. Mark Walker is a recipient of a Canadian Institutes of Health Research Foundation Grant (FDN No. 148438).

Data sharing: The data set for this study is held securely at the prescribed registry BORN Ontario. Data sharing regulations prevent these data from being made available publicly. Enquiries regarding BORN data may be directed to Science@BORNOntario.ca. Please contact Shelley Dougan for more information on accessing BORN data or the study's programming code.

Disclaimer: Parts of this material are based on data and information compiled and provided by BORN Ontario. The analyses, conclusions, opinions and statements expressed herein are solely those of the authors and do not reflect those of the funding or data sources; no endorsement is intended or should be inferred.

Acknowledgements: The authors acknowledge the more than 370000 pregnant people whose prenatal screening experience is reflected in this report, the various Ontario-based laboratories whose data were essential to this work, the birthing hospitals and midwifery practice groups who provide Ontario's birth data and the team at BORN Ontario who ensure that these data are complete and accurate. The authors also thank Mari Teitelbaum for her contribution to the creation of the Prenatal Screening Ontario program.

Accepted: May 21, 2021

Correspondence to: Shelley Dougan, SDougan@BORNOntario.ca 